Article

\title{
Traveling Wave Solutions of a Delayed Cooperative System
}

\author{
Xue-Shi Li ${ }^{1}$ and Shuxia Pan $2, *$ D \\ 1 School of Mathematics, Lanzhou City University, Lanzhou 730070, Gansu, China; lixuesh@yeah.net \\ 2 School of Science, Lanzhou University of Technology, Lanzhou 730050, Gansu, China \\ * Correspondence: shxpan@yeah.net
}

Received: 20 February 2019; Accepted: 12 March 2019; Published: 15 March 2019

check for updates

\begin{abstract}
This paper deals with the dynamics of a delayed cooperative system without quasimonotonicity. Using the contracting rectangles, we obtain a sufficient condition on the stability of the unique positive steady state of the functional differential system. When the spatial domain is whole $\mathbb{R}$, the existence and nonexistence of traveling wave solutions are investigated, during which the asymptotic behavior is investigated by the contracting rectangles.
\end{abstract}

Keywords: contracting rectangle; large delay; minimal wave speed; population dynamics

\section{Introduction}

In population dynamics, there are many important cooperative systems modeling some natural phenomena. For example, some well-known mutualistic interactions are found in a wealth of different environments, such as diatom mats in the ocean, between mangroves and root borers, and between spiders and parasitic wasps [1,2]. In the literature, cooperative systems often lead to monotone dynamics [3-5]. One important cooperative system is the following Lotka-Volterra type system

$$
\left\{\begin{array}{l}
u_{1}^{\prime}(t)=r_{1} u_{1}(t)\left[1-u_{1}(t)+r u_{2}(t)\right] \\
u_{2}^{\prime}(t)=r_{2} u_{2}(t)\left[1-u_{2}(t)+s u_{1}(t)\right]
\end{array}\right.
$$

in which all the parameters are positive. Because Equation (1) is cooperative 2-D ODE system, its dynamics has been fully understood. When the spatial variable is concerned, one model is the following reaction-diffusion system

$$
\left\{\begin{array}{l}
\frac{\partial u_{1}(x, t)}{\partial t}=d_{1} \Delta u_{1}(x, t)+r_{1} u_{1}(x, t)\left[1-u_{1}(x, t)+r u_{2}(x, t)\right] \\
\frac{\partial u_{2}(x, t)}{\partial t}=d_{2} \Delta u_{2}(x, t)+r_{2} u_{2}(x, t)\left[1-u_{2}(x, t)+s u_{1}(x, t)\right]
\end{array}\right.
$$

in which $x \in \mathbb{R}, t>0, d_{1}>0$ and $d_{2}>0$ are diffusive coefficients. We refer to the work of Li et al. [6] and Lin et al. [7] for some results on traveling wave solutions. Moreover, Li et al. [6] and Lin [8] studied the corresponding asymptotic spreading.

However, when it involves time delay, it is possible that it does not generate monotone semiflows, for example

$$
\left\{\begin{array}{l}
u_{1}^{\prime}(t)=r_{1} u_{1}(t)\left[1-u_{1}\left(t-\tau_{1}\right)+r u_{2}\left(t-\tau_{2}\right)\right], \\
u_{2}^{\prime}(t)=r_{2} u_{2}(t)\left[1-u_{2}\left(t-\tau_{3}\right)+s u_{1}\left(t-\tau_{4}\right)\right],
\end{array}\right.
$$

in which $\tau_{1}, \tau_{2}, \tau_{3}, \tau_{4}$ are nonnegative constants. It is evident that Equation (3) is not monotone if $\tau_{1}>0$ or $\tau_{3}>0$. Huang and Zou [9], Li and Wang [10], Li and Lin [11], and Lin et al. [12] studied the existence of traveling wave solutions when $\tau_{1}$ and $\tau_{3}$ are small enough. 
In this paper, we investigate the traveling wave solutions of

$$
\left\{\begin{array}{l}
\frac{\partial u_{1}(x, t)}{\partial t}=d_{1} \Delta u_{1}(x, t)+r_{1} u_{1}(x, t) F_{1}\left(u_{1}, u_{2}\right)(x, t) \\
\frac{\partial u_{2}(x, t)}{\partial t}=d_{2} \Delta u_{2}(x, t)+r_{2} u_{2}(x, t) F_{2}\left(u_{1}, u_{2}\right)(x, t)
\end{array}\right.
$$

where $x \in \mathbb{R}, t>0$ and

$$
\left\{\begin{array}{l}
F_{1}\left(u_{1}, u_{2}\right)(x, t)=1-a_{1} u_{1}(x, t)-b_{1} \int_{-\tau}^{0} u_{1}(x, t+s) d \eta_{11}(s)+e_{1} \int_{-\tau}^{0} u_{2}(x, t+s) d \eta_{12}(s), \\
F_{2}\left(u_{1}, u_{2}\right)(x, t)=1-a_{2} u_{2}(x, t)-b_{2} \int_{-\tau}^{0} u_{2}(x, t+s) d \eta_{22}(s)+e_{2} \int_{-\tau}^{0} u_{1}(x, t+s) d \eta_{21}(s),
\end{array}\right.
$$

in which $r_{1}>0, r_{2}>0$, and $a_{i}, b_{i}, c_{i}, i=1,2$, are nonnegative constants satisfying

$$
a_{1} a_{2}>e_{1} e_{2}
$$

and $\tau>0$ such that

$$
\eta_{i j}(s) \text { is nondecreasing on }[-\tau, 0] \text { and } \eta_{i j}(0)-\eta_{i j}(-\tau)=1, i, j=1,2 \text {. }
$$

Clearly, Equation (4) has a positive spatial homogeneous steady state formulated by

$$
K=\left(k_{1}, k_{2}\right)=\left(\frac{e_{2}+a_{1}+b_{1}}{\left(a_{1}+b_{1}\right)\left(a_{2}+b_{2}\right)-e_{1} e_{2}}, \frac{e_{1}+a_{2}+b_{2}}{\left(a_{1}+b_{1}\right)\left(a_{2}+b_{2}\right)-e_{1} e_{2}}\right)
$$

if $\left(a_{1}+b_{1}\right)\left(a_{2}+b_{2}\right)>e_{1} e_{2}$ which is implied by Equation (6). Moreover, $0=(0,0)$ is a trivial spatial homogeneous steady state.

It is clear that Equation (4) may not be a quasimonotone system, although its corresponding undelayed system is a cooperative system (see Li et al. [6]). At the same time, Equation (4) does not satisfy the local quasimonotonicity in $[13,14]$. Thus, it is difficult to study it by constructing two auxiliary quasimonotone systems. Of course, besides those in [13-16], there are also some results for delayed nonmonotone model with large delay. For example, if $e_{1}<0, e_{2}<0$ in Equation (4), then Martin and Smith [17] and Smith [5] gave some results on the stability of steady states. Lin and Ruan [18] further studied the existence and nonexistence of traveling wave solutions. Very recently, Meng et al. [19] investigated the monotone traveling wave solutions of Equation (4) if the intraspecific delay is small, which leads to the quasimonotonicity in the sense of exponential ordering [5]. Besides the traveling wave solutions, there are also some other features of entire solutions formulating by wave type solutions (see [20-22] for some examples of nonmonotone equations).

In this paper, by the ideas in $[17,18]$, we study the dynamics of Equation (4). We first investigate the stability of the following initial value problem

$$
\left\{\begin{array}{l}
\frac{d u_{1}(t)}{d t}=r_{1} u_{1}(t)\left[1-a_{1} u_{1}(t)-b_{1} \int_{-\tau}^{0} u_{1}(t+s) d \eta_{11}(s)+e_{1} \int_{-\tau}^{0} u_{2}(t+s) d \eta_{12}(s)\right. \\
\frac{d u_{2}(t)}{d t}=r_{2} u_{2}(t)\left[1-a_{2} u_{2}(t)-b_{2} \int_{-\tau}^{0} u_{2}(t+s) d \eta_{22}(s)+e_{2} \int_{-\tau}^{0} u_{1}(t+s) d \eta_{21}(s)\right] \\
u_{i}(\theta)=\phi_{i}(\theta), \theta \in[-\tau, 0], i=1,2
\end{array}\right.
$$

in which all the parameters are the same as those in Equation (4) and $\phi_{i}(\theta), i=1,2$, are continuous for $\theta \in[-\tau, 0]$. In fact, the stability was obtained by the authors of [23,24], and we present the result for the sake of verifying the asymptotic behavior of traveling wave solutions. Then, the existence and nonexistence of invasion traveling wave solutions of Equation (4) are considered in Section 3. More precisely, we give the existence of traveling wave solutions by constructing upper and lower solutions, investigate the asymptotic behavior by applying the contracting rectangles, and confirm the nonexistence of traveling wave solutions by utilizing the theory of asymptotic spreading and constructing an auxiliary equation. 


\section{Stability of Positive Steady States}

In this paper, we use the standard partial ordering in $\mathbb{R}^{2}$. That is, for $u=\left(u_{1}, u_{2}\right), v=\left(v_{1}, v_{2}\right) \in \mathbb{R}^{2}$, we write $u \geq v$ provided $u_{i} \geq v_{i}$ for $i=1,2, u>v$ provided $u \geq v$ but $u \neq v$, and $u \gg v$ provided $u_{1}>v_{1}$ and $u_{2}>v_{2}$.

To investigate Equation (7), we first introduce the following quasimonotone system

$$
\left\{\begin{array}{l}
\frac{d \bar{u}_{1}(t)}{d t}=r_{1} \bar{u}_{1}(t)\left[1-a_{1} \bar{u}_{1}(t)+e_{1} \int_{-\tau}^{0} \bar{u}_{2}(t+s) d \eta_{12}(s)\right] \\
\frac{d \bar{u}_{2}(t)}{d t}=r_{1} \bar{u}_{2}(t)\left[1-a_{2} \bar{u}_{2}(t)+e_{2} \int_{-\tau}^{0} \bar{u}_{1}(t+s) d \eta_{21}(s)\right] \\
\bar{u}_{i}(\theta)=\bar{\phi}_{i}(\theta), \theta \in[-\tau, 0], i=1,2
\end{array}\right.
$$

where $\bar{\phi}_{i}(\theta), i=1,2$, are continuous for $\theta \in[-\tau, 0]$.

If Equation (6) holds, then Equation (8) admits a spatially homogeneous steady state as follows

$$
\bar{K}=\left(\bar{k}_{1}, \bar{k}_{2}\right)=\left(\frac{e_{2}+a_{1}}{a_{1} a_{2}-e_{1} e_{2}}, \frac{e_{1}+a_{2}}{a_{1} a_{2}-e_{1} e_{2}}\right) .
$$

Evidently, $\left.K\right|_{b_{1}=b_{2}=0}=\bar{K}$. Moreover, since

$$
\begin{aligned}
& \frac{d}{d b_{1}}\left[\frac{e_{2}+a_{1}+b_{1}}{\left(a_{1}+b_{1}\right)\left(a_{2}+b_{2}\right)-e_{1} e_{2}}\right] \\
= & \frac{\left(a_{1}+b_{1}\right)\left(a_{2}+b_{2}\right)-e_{1} e_{2}-\left(e_{2}+a_{1}+b_{1}\right)\left(a_{2}+b_{2}\right)}{\left(\left(a_{1}+b_{1}\right)\left(a_{2}+b_{2}\right)-e_{1} e_{2}\right)^{2}} \\
= & \frac{-e_{1} e_{2}-e_{2}\left(a_{2}+b_{2}\right)}{\left(\left(a_{1}+b_{1}\right)\left(a_{2}+b_{2}\right)-e_{1} e_{2}\right)^{2}} \leq 0,
\end{aligned}
$$

and

$$
\frac{d}{d b_{2}}\left[\frac{e_{1}+a_{2}+b_{2}}{\left(a_{1}+b_{1}\right)\left(a_{2}+b_{2}\right)-e_{1} e_{2}}\right] \leq 0,
$$

and $b_{1} \geq 0, b_{2} \geq 0$, we have proved $\bar{k}_{i} \geq k_{i}, i=1,2$.

The existence and uniqueness of mild solution of Equations (7) and (8) can be obtained by the theory of functional differential equations (see Hale and Verduyn Lunel [25]) and we omit it here. By the classical theory of classical monotone dynamics systems (see Smith [5]), the following result for Equation (8) is clear.

Lemma 1. Assume that Equation (6) holds. If $\bar{\phi}_{i}(\theta) \geq 0, \theta \in[-\tau, 0]$ and $\bar{\phi}_{i}(0)>0$ for $i=1,2$, then the unique mild solution $\left(u_{1}(t), u_{2}(t)\right)$ to Equation (8) satisfies

$$
\bar{u}_{i}(t) \rightarrow \bar{k}_{i}, t \rightarrow \infty, i=1,2
$$

Using the quasipositivity (see Smith [5], Theorem 5.2.1), we can obtain the following conclusion on the positivity of mild solution to Equation (7).

Lemma 2. Assume that $\phi_{i}(\theta) \geq 0$ for $\theta \in[-\tau, 0], i=1,2$. Then,

$$
u_{i}(t) \geq 0, i=1,2, t \in\left(0, t_{1}\right),
$$

in which $t_{1}$ (may be infinite) is the maximal interval of the existence of Equation (7).

From Lemma 2, if $\bar{\phi}_{i}(\theta) \geq \phi_{i}(\theta) \geq 0, i=1,2, \theta \in[-\tau, 0]$, then $\left(u_{1}(t), u_{2}(t)\right)$ becomes a sub solution of Equation (8) satisfying quasimonotonicity. By the standard comparison principle 
of functional differential equations with quasimonotonicity $[17,26]$, the following conclusion can be obtained.

Lemma 3. Assume that Equation (6) holds. If $\bar{\phi}_{i}(\theta) \geq 0$ for $\theta \in[-\tau, 0], i=1,2$, then Equation (8) admits a unique bounded mild solution $\left(\bar{u}_{1}(t), \bar{u}_{2}(t)\right)$ for $t>0$. Moreover,

$$
0 \leq \liminf _{t \rightarrow \infty} \bar{u}_{i}(t) \leq \limsup _{t \rightarrow \infty} \bar{u}_{i}(t) \leq \bar{k}_{i}, i=1,2
$$

Let $\left(u_{1}(t), u_{2}(t)\right)$ be the unique mild solution of Equation (7) with $\bar{\phi}_{i}(\theta) \geq \phi_{i}(\theta) \geq 0$ for $\theta \in[-\tau, 0], i=$ 1,2 , then

$$
u_{i}(t) \leq \bar{u}_{i}(t), i=1,2, t \geq 0
$$

and

$$
0 \leq \liminf _{t \rightarrow \infty} u_{i}(t) \leq \limsup _{t \rightarrow \infty} u_{i}(t) \leq \bar{k}_{i}, i=1,2
$$

We now present our main result of this section.

Theorem 1. Assume that $\phi_{i}(\theta) \geq 0, \phi_{i}(0)>0$ for all $\theta \in[-\tau, 0], i=1$, 2. If $b_{1} \bar{k}_{1}<1$ and $b_{2} \bar{k}_{2}<1$ hold, then the unique mild solution $\left(u_{1}(t), u_{2}(t)\right)$ of Equation (7) satisfies

$$
u_{i}(t) \rightarrow k_{i}, t \rightarrow \infty, i=1,2
$$

Proof. We prove it using the method by Smith [5]. For $s \in[0,1]$, define $E(s)$ and $F(s)$ as follows

$$
E(s)=\left(s k_{1}, s k_{2}\right), F(s)=\left(s k_{1}+(1-s)\left(\bar{k}_{1}+\epsilon_{1}\right), s k_{2}+(1-s)\left(\bar{k}_{2}+\epsilon_{2}\right)\right),
$$

in which $\epsilon_{1}>, \epsilon_{2}>0$ such that

$$
b_{1}\left(\bar{k}_{1}+\epsilon_{1}\right)<1, b_{2}\left(\bar{k}_{2}+\epsilon_{2}\right)<1
$$

and

$$
e_{1} \epsilon_{2}<a_{1} \epsilon_{1}, e_{2} \epsilon_{1}<a_{2} \epsilon_{2}
$$

By Equation (6), they are admissible.

Assume that $\left(u_{1}(t+\theta), u_{2}(t+\theta)\right) \in[E(s), F(s)]$ for all $\theta \in[-\tau, 0], t \geq 0$ and some $s \in(0,1)$. If $u_{1}(t)=s k_{1}$, then $\int_{-\tau}^{0} d \eta_{11}(s)=\int_{-\tau}^{0} d \eta_{12}(s)=1$ and

$$
a_{1} k_{1}+b_{1} k_{1}-e_{1} k_{2}=1
$$

such that

$$
\begin{aligned}
& 1-a_{1} u_{1}(t)-b_{1} \int_{-\tau}^{0} u_{1}(t+s) d \eta_{11}(s)+e_{1} \int_{-\tau}^{0} u_{2}(t+s) d \eta_{12}(s) \\
\geq & 1-a_{1} u_{1}(t)-b_{1}\left(s k_{1}+(1-s)\left(\bar{k}_{1}+\epsilon_{1}\right)\right)+e_{1} s k_{2} \\
= & 1-a_{1} s k_{1}-b_{1}\left(s k_{1}+(1-s)\left(\bar{k}_{1}+\epsilon_{1}\right)\right)+e_{1} s k_{2} \\
= & 1-s-b_{1}(1-s)\left(\bar{k}_{1}+\epsilon_{1}\right) \\
= & (1-s)\left[1-b_{1}\left(\bar{k}_{1}+\epsilon_{1}\right)\right] \\
> & 0
\end{aligned}
$$


from the definition of $\epsilon_{1}$. Similarly, if $\left(u_{1}(t+\theta), u_{2}(t+\theta)\right) \in[E(s), F(s)]$ and $u_{2}(t)=s k_{2}$ with $\theta \in$ $[-\tau, 0], s \in(0,1)$ and $t \geq 0$, then

$$
1-a_{2} u_{2}(t)-b_{2} \int_{-\tau}^{0} u_{2}(t+s) d \eta_{22}(s)+e_{2} \int_{-\tau}^{0} u_{1}(t+s) d \eta_{21}(s)>0
$$

Moreover, when $\left(u_{1}(t+\theta), u_{2}(t+\theta)\right) \in[E(s), F(s)]$ with $\theta \in[-\tau, 0], t \geq 0$ and some $s \in[0,1)$, if $u_{1}(t)=s k_{1}+(1-s)\left(\bar{k}_{1}+\epsilon_{1}\right)$, then

$$
\begin{aligned}
& 1-a_{1} u_{1}(t)-b_{1} \int_{-\tau}^{0} u_{1}(t+s) d \eta_{11}(s)+e_{1} \int_{-\tau}^{0} u_{2}(t+s) d \eta_{12}(s) \\
\leq & 1-a_{1} u_{1}(t)-b_{1} s k_{1}+e_{1}\left(s k_{2}+(1-s)\left(\bar{k}_{2}+\epsilon_{2}\right)\right) \\
= & 1-a_{1}\left(s k_{1}+(1-s)\left(\bar{k}_{1}+\epsilon_{1}\right)\right)-b_{1} s k_{1}+e_{1}\left(s k_{2}+(1-s)\left(\bar{k}_{2}+\epsilon_{2}\right)\right) \\
= & (1-s)\left[1-a_{1}\left(\bar{k}_{1}+\epsilon_{1}\right)+e_{1}\left(\bar{k}_{2}+\epsilon_{2}\right)\right] \\
< & 0 .
\end{aligned}
$$

Similarly, we have

$$
1-a_{2} u_{2}(t)-b_{2} \int_{-\tau}^{0} u_{2}(t+s) d \eta_{22}(s)+e_{2} \int_{-\tau}^{0} u_{1}(t+s) d \eta_{21}(s)<0
$$

when $\left(u_{1}(t+\theta), u_{2}(t+\theta)\right) \in[E(s), F(s)]$ with $\theta \in[-\tau, 0]$, and $u_{2}(t)=s k_{2}+(1-s)\left(\bar{k}_{2}+\epsilon_{2}\right)$ with $s \in[0,1), t \geq 0$.

Moreover, from Lemma 3, we also see that

$$
\liminf _{t \rightarrow \infty} u_{i}(t) \geq\left(1-b_{i} \bar{k}_{i}\right) / a_{i}>0, i=1,2
$$

Let $s_{0}>0$ be small such that

$$
s_{0} k_{i}<\left(1-b_{i} \bar{k}_{i}\right) / a_{i}, s_{0} k_{i}+\left(1-s_{0}\right)\left(\bar{k}_{i}+\epsilon_{i}\right)>\bar{k}_{i}
$$

Then, it implies that there exists $T>0$ such that

$$
E\left(s_{0}\right)<\left(u_{1}(t), u_{2}(t)\right)<F\left(s_{0}\right), t>T .
$$

Define

$$
\bar{u}_{i}=\limsup _{t \rightarrow \infty} u_{i}(t), \underline{u}_{i}=\liminf _{t \rightarrow \infty} u_{i}(t), i=1,2,
$$

then they are positive constants by Equation (10). If $\bar{u}_{i}=\underline{u}_{i}=k_{i}$, then the conclusion is true. Otherwise, there exists $s^{\prime} \in\left(s_{0}, 1\right)$ such that

$$
s^{\prime}=\sup \left\{s: E(s)<\left(u_{1}(t), u_{2}(t)\right)<F(s)\right\},
$$

which implies that at least one of the following is true

$$
\underline{u}_{i}=s^{\prime} k_{i}, \bar{u}_{i}=s^{\prime} k_{i}+\left(1-s^{\prime}\right)\left(\bar{k}_{i}+\epsilon_{i}\right), i=1,2 .
$$

If $\underline{u}_{1}=s^{\prime} k_{1}$, then there exists $\left\{t_{n}\right\}$ with $t_{n} \rightarrow \infty, n \rightarrow \infty$ such that

$$
u\left(t_{n}\right) \rightarrow s^{\prime} k_{1}, u^{\prime}\left(t_{n}\right) \rightarrow 0, n \rightarrow \infty
$$


and

$$
\begin{aligned}
& \liminf _{t \rightarrow \infty}\left[1-a_{1} u_{1}(t)-b_{1} \int_{-\tau}^{0} u_{1}(t+s) d \eta_{11}(s)+e_{1} \int_{-\tau}^{0} u_{2}(t+s) d \eta_{12}(s)\right] \\
\geq & 1-a_{1} s^{\prime} k_{1}-b_{1}\left(s^{\prime} k_{1}+\left(1-s^{\prime}\right)\left(\bar{k}_{1}+\epsilon_{1}\right)\right)+e_{1} s^{\prime} k_{2} \\
= & 1-s^{\prime}-b_{1}\left(1-s^{\prime}\right)\left(\bar{k}_{1}+\epsilon_{1}\right) \\
= & \left(1-s^{\prime}\right)\left[1-b_{1}\left(\bar{k}_{1}+\epsilon_{1}\right)\right] \\
> & 0
\end{aligned}
$$

by Equation (9). A contradiction occurs. In a similar way, we can confirm that $s^{\prime}<1$ is impossible. The proof is complete.

Remark 1. By the method of Smith [5], $[E(s), F(s)]$ is a contracting rectangle of Equation (7).

\section{Traveling Wave Solutions}

In this section, we consider the traveling wave solutions of Equation (4), throughout which Equation (6) holds. We first give the following definition.

Definition 1. A traveling wave solution of Equation (4) is a special solution with the form

$$
u_{i}(x, t)=\varphi_{i}(x+c t), i=1,2,
$$

in which $c>0$ is the wave speed while $\left(\varphi_{1}(\xi), \varphi_{2}(\xi)\right) \in C^{2}\left(\mathbb{R}, \mathbb{R}^{2}\right)$ is the wave profile.

By the definition, $\left(\varphi_{1}(\xi), \varphi_{2}(\xi)\right)$ must satisfy the following functional differential system

$$
\left\{\begin{array}{l}
d_{1} \varphi_{1}^{\prime \prime}(\xi)-c \varphi_{1}^{\prime}(\xi)+H_{1}\left(\varphi_{1}, \varphi_{2}\right)(\xi)=0 \\
d_{2} \varphi_{2}^{\prime \prime}(\xi)-c \varphi_{2}^{\prime}(\xi)+H_{2}\left(\varphi_{1}, \varphi_{2}\right)(\xi)=0
\end{array}\right.
$$

with

$$
\left\{\begin{array}{l}
H_{1}\left(\varphi_{1}, \varphi_{2}\right)(\xi)=r_{1} \varphi_{1}(\xi)\left[1-a_{1} \varphi_{1}(\xi)-b_{1} \int_{-\tau}^{0} \varphi_{1}(\xi+c s) d \eta_{11}(s)+e_{1} \int_{-\tau}^{0} \varphi_{2}(\xi+c s) d \eta_{12}(s)\right] \\
H_{2}\left(\varphi_{1}, \varphi_{2}\right)(\xi)=r_{2} \varphi_{2}(\xi)\left[1-a_{2} \varphi_{2}(\xi)-b_{2} \int_{-\tau}^{0} \varphi_{2}(\xi+c s) d \eta_{22}(s)+e_{2} \int_{-\tau}^{0} \varphi_{1}(\xi+c s) d \eta_{21}(s)\right] .
\end{array}\right.
$$

In particular, we also consider the positive traveling wave solutions of Equation (4) formulating the invasion of two cooperative species, which can be characterized by the following asymptotic boundary conditions

$$
\lim _{\xi \rightarrow-\infty} \varphi_{i}(\xi)=0, \lim _{\xi \rightarrow \infty} \varphi_{i}(\xi)=k_{i}, i=1,2 .
$$

When the wave speed is small, we have the following result on the nonexistence of traveling wave solutions.

Theorem 2. If $c<\max \left\{2 \sqrt{d_{1} r_{1}}, 2 \sqrt{d_{2} r_{2}}\right\}$, then Equation (11) has no bounded positive solution satisfying

$$
\lim _{\xi \rightarrow-\infty}\left(\varphi_{1}(\xi), \varphi_{2}(\xi)\right)=(0,0), \liminf _{\xi \rightarrow \infty} \varphi_{i}(\xi)>0, i=1,2, \xi \in \mathbb{R}
$$

Proof. It suffices to study the case of $d_{1} r_{1} \geq d_{2} r_{2}$. Were the statement false, then there exists $c^{\prime}<2 \sqrt{d_{1} r_{1}}$ such that Equation (11) with $c=c^{\prime}$ has a positive solution $\left(\varphi_{1}(\xi), \varphi_{2}(\xi)\right)$ satisfying Equation (13). Let

$$
2 \sqrt{d_{1} r_{1}(1-4 \epsilon)}=c^{\prime}
$$


then there exists $M>0$ large (but finite) such that

$$
d_{1} \varphi_{1}^{\prime \prime}(\xi)-c^{\prime} \varphi_{1}^{\prime}(\xi)+r_{1} \varphi_{1}(\xi)\left[1-\epsilon-M \varphi_{1}(\xi)\right] \leq 0 .
$$

If fact, by Equation (13), there exists $\xi_{0}<0$ such that

$$
b_{1} \int_{-\tau}^{0} \varphi_{1}(\xi+c s) d \eta_{11}(s)<\epsilon, \xi<\xi_{0}
$$

Define $\varepsilon=\inf _{\tilde{\zeta} \geq \xi_{0}} \varphi_{1}(\xi)$, then Equation (13) indicates $\varepsilon>0$. Let

$$
\left(M-a_{1}\right) \varepsilon=b_{1} \sup _{\xi \in \mathbb{R}} \varphi_{1}(\xi)
$$

and we have proved what we wanted.

By the definition $u_{1}(x, t)=\varphi_{1}\left(x+c^{\prime} t\right)$, then it satisfies

$$
\left\{\begin{array}{l}
\frac{\partial u(x, t)}{\partial t} \geq d_{1} \Delta u(x, t)+r_{1} u_{1}(x, t)\left[1-\epsilon-M u_{1}(x, t)\right] \\
u_{1}(x, 0)=\varphi_{1}(x)>0
\end{array}\right.
$$

Using the theory of asymptotic spreading [27], we see that

$$
\lim _{t \rightarrow \infty} \inf _{|x| \leq c_{1} t} u_{1}(x, t)>\frac{1-\epsilon}{M}
$$

in which $c_{1}=2 \sqrt{d_{1} r_{1}(1-2 \epsilon)}>c^{\prime}$. Let $-2 x=\left(c_{1}+c^{\prime}\right) t$, then a contradiction occurs between Equations (13) and (14) when $t \rightarrow \infty$. The proof is complete.

When $c>\max \left\{2 \sqrt{d_{1} r_{1}}, 2 \sqrt{d_{2} r_{2}}\right\}$, we define

$$
\gamma_{i}=\frac{c-\sqrt{c^{2}-4 d_{i} r_{i}}}{2 d_{i}}, \gamma_{2+i}=\frac{c+\sqrt{c^{2}-4 d_{i} r_{i}}}{2 d_{i}}, i=1,2
$$

Further define

$$
\Gamma=\left(\gamma_{1}, \gamma_{3}\right) \bigcap\left(\gamma_{2}, \gamma_{4}\right) .
$$

Then, there exists $c_{0} \geq \max \left\{2 \sqrt{d_{1} r_{1}}, 2 \sqrt{d_{2} r_{2}}\right\}$ such that $c>c_{0}$ implies that $\Gamma$ is nonempty.

Assume that $c>c_{0}$, then we can choose $\gamma \in \Gamma$. Define continuous functions

$$
\bar{\varphi}_{i}(\xi)=\min \left\{e^{\gamma_{i}} \tilde{\xi}+q \bar{k}_{i} e^{\gamma \zeta}, \bar{k}_{i}\right\}, i=1,2
$$

in which $q>1$ is a positive constant clarified later. Moreover, select $\gamma_{5}, \gamma_{6}$ such that

$$
\gamma_{5} \in\left(\gamma_{1}, \min \left\{2 \gamma_{1}, \gamma_{3}, \gamma_{1}+\gamma_{2}\right\}\right), \gamma_{6} \in\left(\gamma_{2}, \min \left\{2 \gamma_{2}, \gamma_{4}, \gamma_{1}+\gamma_{2}\right\}\right)
$$

Construct continuous functions as follows

$$
\underline{\varphi}_{i}(\xi)=\max \left\{e^{\gamma_{i}} \xi-p e^{\gamma_{4+i} \xi}, 0\right\}, i=1,2,
$$

where $p>1$ is a positive constant formulated later.

Lemma 4. Assume that $c>c_{0}$. Then, there exist $p, q$ such that

$$
\left\{\begin{array}{l}
d_{i} \bar{\varphi}_{i}^{\prime \prime}(\xi)-c \bar{\varphi}_{i}^{\prime}(\xi)+\bar{H}_{i}(\xi) \leq 0, e^{\gamma_{i}} \bar{\xi}+q \bar{k}_{i} e^{\gamma \xi} \neq \bar{k}_{i} \\
d_{i} \underline{\varphi}_{i}^{\prime \prime}(\xi)-c \underline{\varphi}_{i}^{\prime}(\xi)+\underline{H}_{i}(\xi) \geq 0, e^{\gamma_{i} \xi}-p e^{\gamma_{4+i} \xi} \neq 0
\end{array}\right.
$$


in which

$$
\begin{aligned}
& \bar{H}_{1}(\xi)=r_{1} \bar{\varphi}_{1}(\xi)\left[1-a_{1} \bar{\varphi}_{1}(\xi)-b_{1} \int_{-\tau}^{0} \underline{\varphi}_{1}(\xi+c s) d \eta_{11}(s)+e_{1} \int_{-\tau}^{0} \bar{\varphi}_{2}(\xi+c s) d \eta_{12}(s)\right], \\
& \bar{H}_{2}(\xi)=r_{2} \bar{\varphi}_{2}(\xi)\left[1-a_{2} \bar{\varphi}_{2}(\xi)-b_{2} \int_{-\tau}^{0} \underline{\varphi}_{2}(\xi+c s) d \eta_{22}(s)+e_{2} \int_{-\tau}^{0} \bar{\varphi}_{1}(\xi+c s) d \eta_{21}(s)\right], \\
& \underline{H}_{1}(\xi)=r_{1} \underline{\varphi}_{1}(\xi)\left[1-a_{1} \underline{\varphi}_{1}(\xi)-b_{1} \int_{-\tau}^{0} \bar{\varphi}_{1}(\xi+c s) d \eta_{11}(s)+e_{1} \int_{-\tau}^{0} \underline{\varphi}_{2}(\xi+c s) d \eta_{12}(s)\right], \\
& \underline{H}_{2}(\xi)=r_{2} \underline{\varphi}_{2}(\xi)\left[1-a_{2} \underline{\varphi}_{2}(\xi)-b_{2} \int_{-\tau}^{0} \bar{\varphi}_{2}(\xi+c s) d \eta_{22}(s)+e_{2} \int_{-\tau}^{0} \underline{\varphi}_{1}(\xi+c s) d \eta_{21}(s)\right] .
\end{aligned}
$$

Proof. We first verify the inequality on $\bar{\varphi}_{1}(\xi)$. If $\bar{\varphi}_{1}(\xi)=\bar{k}_{1}$, then the result is clear. Otherwise, we have

$$
\begin{aligned}
\bar{H}_{1}(\xi) & \leq r_{1} \bar{\varphi}_{1}(\xi)\left[1-a_{1} \bar{\varphi}_{1}(\xi)+e_{1} \int_{-\tau}^{0} \bar{\varphi}_{2}(\xi+c s) d \eta_{12}(s)\right] \\
& \leq r_{1} \bar{\varphi}_{1}(\xi)\left[1-a_{1}\left(e^{\gamma_{1} \xi}+q \bar{k}_{1} e^{\gamma \xi}\right)+e_{1}\left(e^{\gamma_{2} \xi}+q \bar{k}_{2} e^{\gamma \tau}\right)\right] \\
& <r_{1} \bar{\varphi}_{1}(\xi)\left[1-a_{1} e^{\gamma_{1} \xi}+e_{1} e^{\gamma_{2} \xi}\right] \\
& <r_{1} \bar{\varphi}_{1}(\xi)\left[1+e_{1} e^{\gamma_{2} \xi}\right]
\end{aligned}
$$

which also implies that

$$
\begin{aligned}
& d_{1} \bar{\varphi}_{1}^{\prime \prime}(\xi)-c \bar{\varphi}_{1}^{\prime}(\xi)+\bar{H}_{1}(\xi) \\
\leq & \left(d_{1} \gamma_{1}^{2}-c \gamma_{1}+r_{1}\right) e^{\gamma_{1} \xi} \\
& +q \bar{k}_{1} e^{\gamma \tilde{\zeta}}\left(d_{1} \gamma^{2}-c \gamma+r_{1}+e_{1} e^{\gamma_{2} \xi}+e_{1} e^{\left(\gamma_{1}+\gamma_{2}-\gamma\right) \xi}\right) .
\end{aligned}
$$

Let $q>1$ be large enough such that $e^{\gamma_{2} \xi}+q \bar{k}_{1} e^{\gamma \xi}>1$ implies $-\xi>0$ is large and

$$
d_{1} \gamma^{2}-c \gamma+r_{1}+e_{1} e^{\gamma_{2} \xi}+e_{1} e^{\left(\gamma_{1}+\gamma_{2}-\gamma\right) \xi}<0
$$

Then,

$$
d_{1} \bar{\varphi}_{1}^{\prime \prime}(\xi)-c \bar{\varphi}_{1}^{\prime}(\xi)+\bar{H}_{1}(\xi) \leq 0
$$

Similarly, if $q>1$ such that

$$
d_{2} \gamma^{2}-c \gamma+r_{2}+e_{2} e^{\gamma_{1} \tilde{\xi}}+e_{2} e^{\left(\gamma_{1}+\gamma_{2}-\gamma\right) \xi}<0,
$$

then

$$
d_{2} \bar{\varphi}_{2}^{\prime \prime}(\xi)-c \bar{\varphi}_{2}^{\prime}(\xi)+\bar{H}_{2}(\xi) \leq 0 .
$$

To continue our discussion, we fix $q>1$ such that Equations (15) and (16) hold.

We now prove the following inequality

$$
d_{1} \underline{\varphi}_{1}^{\prime \prime}(\xi)-c \underline{\varphi}_{1}^{\prime}(\xi)+\underline{H}_{1}(\xi) \geq 0,
$$

and the result is clear if $\underline{\varphi}_{1}(\xi)=0$. Otherwise, we first choose $p_{1}>1$ such that $p>p_{1}$ indicates that

$$
\bar{\varphi}_{1}(\xi) \leq 2 e^{\gamma_{1} \xi}, \bar{\varphi}_{2}(\xi) \leq 2 e^{\gamma_{2} \xi}
$$


if $e^{\gamma_{1} \xi} \geq p e^{\gamma_{5} \xi}$ or $e^{\gamma_{2} \xi} \geq p e^{\gamma_{6} \xi}$. Then

$$
\begin{aligned}
\underline{H}_{1}(\xi) & =r_{1} \underline{\varphi}_{1}(\xi)\left[1-a_{1} \underline{\varphi}_{1}(\xi)-b_{1} \int_{-\tau}^{0} \bar{\varphi}_{1}(\xi+c s) d \eta_{11}(s)+e_{1} \int_{-\tau}^{0} \underline{\varphi}_{2}(\xi+c s) d \eta_{12}(s)\right] \\
& \geq r_{1} \underline{\varphi}_{1}(\xi)\left[1-a_{1} \underline{\varphi}_{1}(\xi)-b_{1} \int_{-\tau}^{0} \bar{\varphi}_{1}(\xi+c s) d \eta_{11}(s)\right] \\
& \geq r_{1} \underline{\varphi}_{1}(\xi)\left[1-a_{1} \underline{\varphi}_{1}(\xi)-2 b_{1} e^{\gamma_{1} \xi}\right] \\
& \geq r_{1} \underline{\varphi}_{1}(\xi)\left[1-\left(a_{1}+2 b_{1}\right) e^{\gamma_{1} \xi}\right] \\
& \geq r_{1} \underline{\varphi}_{1}(\xi)-\left(a_{1}+2 b_{1}\right) e^{2 \gamma_{1} \xi} .
\end{aligned}
$$

Furthermore, we have

$$
\begin{aligned}
& d_{1} \underline{\varphi}_{1}^{\prime \prime}(\xi)-c \underline{\varphi}_{1}^{\prime}(\xi)+\underline{H}_{1}(\xi) \\
\geq & \left(d_{1} \gamma_{5}^{2}-c \gamma_{5}+r_{1}\right) p e^{\gamma_{5} \xi}-\left(a_{1}+2 b_{1}\right) e^{2 \gamma_{1} \xi} .
\end{aligned}
$$

Choosing $p>p_{1}$ and

$$
p>1-\frac{a_{1}+2 b_{1}}{d_{1} \gamma_{5}^{2}-c \gamma_{5}+r_{1}}>1
$$

we have obtained

$$
d_{1} \underline{\varphi}_{1}^{\prime \prime}(\xi)-c \underline{\varphi}_{1}^{\prime}(\xi)+\underline{H}_{1}(\xi) \geq 0
$$

Similarly, if

$$
p>p_{1}-\frac{a_{1}+2 b_{1}}{d_{1} \gamma_{5}^{2}-c \gamma_{5}+r_{1}}-\frac{a_{2}+2 b_{2}}{d_{2} \gamma_{6}^{2}-c \gamma_{6}+r_{2}}>1
$$

then

$$
d_{2} \underline{\varphi}_{2}^{\prime \prime}(\xi)-c \underline{\varphi}_{2}^{\prime}(\xi)+\underline{H}_{2}(\xi) \geq 0
$$

The proof is complete.

Remark 2. For any fixed $c$, we can first choose $q$, then $p$.

Lemma 5. Assume that $c>c_{0}$. Then, Equation (11) has a strictly positive solution.

Proof. We now prove the result by Schauder's fixed point theorem. Throughout the proof, we assume that $c$ is a fixed constant. Let $\beta>0$ such that

$$
\beta u+r_{1} u\left[1-a_{1} u-b_{1} \bar{k}_{1}\right], \beta v+r_{2} v\left[1-a_{2} v-b_{2} \bar{k}_{2}\right]
$$

are monotone increasing in $u \in\left[0, \bar{k}_{1}\right], v \in\left[0, \bar{k}_{2}\right]$, respectively. Further, define

$$
\begin{array}{ll}
\lambda_{1}=\frac{c-\sqrt{c^{2}+4 \beta d_{1}}}{2 d_{1}}, & \lambda_{2}=\frac{c+\sqrt{c^{2}+4 \beta d_{1}}}{2 d_{1}}, \\
\lambda_{3}=\frac{c-\sqrt{c^{2}+4 \beta d_{2}}}{2 d_{2}}, & \lambda_{4}=\frac{c+\sqrt{c^{2}+4 \beta d_{2}}}{2 d_{2}} .
\end{array}
$$

Let $X$ be the following functional space

$$
X=\left\{\mathbf{u}: \mathbf{u} \text { is a bounded and uniformly continuous function from } \mathbb{R} \text { to } \mathbb{R}^{2}\right\} .
$$


If $\mathbf{a}, \mathbf{b} \in \mathbb{R}^{2}$ with $\mathbf{a} \leq \mathbf{b}$, then $X_{[\mathbf{a}, \mathbf{b}]}$ is defined by

$$
X_{[\mathbf{a}, \mathbf{b}]}=\{\mathbf{u} \in X: \mathbf{a} \leq \mathbf{u}(\xi) \leq \mathbf{b}, \xi \in \mathbb{R}\} .
$$

Let $4 \mu=\min \left\{-\lambda_{1},-\lambda_{3}\right\}$. Define

$$
B_{\mu}\left(\mathbb{R}, \mathbb{R}^{2}\right)=\left\{\mathbf{u} \in X: \sup _{\xi \in \mathbb{R}}\|\mathbf{u}(\xi)\| e^{-\mu|\xi|}<\infty\right\}
$$

and

$$
|\mathbf{u}|_{\mu}=\sup _{\xi \in \mathbb{R}}\left\{\|\mathbf{u}(\xi)\| e^{-\mu|\xi|}\right\}
$$

then $B_{\mu}\left(\mathbb{R}, \mathbb{R}^{2}\right)$ is a Banach space with the decay norm $|\cdot|_{\mu}$, where $\|\cdot\|$ denotes the standard supremum norm in $\mathbb{R}^{2}$.

We define

$$
\Sigma=\left\{\left(\varphi_{1}, \varphi_{2}\right) \in X:\left(\underline{\varphi}_{1} \underline{\varphi}_{2}\right) \leq\left(\varphi_{1}, \varphi_{2}\right) \leq\left(\bar{\varphi}_{1}, \bar{\varphi}_{2}\right)\right\} .
$$

Then, $\Sigma$ is nonempty, convex. It is also bounded and closed in the sense of the decay norm $|\cdot|_{\mu}$. Moreover, if $\left(\varphi_{1}, \varphi_{2}\right) \in \Sigma$, we define $P=\left(P_{1}, P_{2}\right): \Sigma \rightarrow X$ as follows

$$
\left\{\begin{array}{l}
P_{1}\left(\varphi_{1}, \varphi_{2}\right)(\xi)=\frac{1}{d_{1}\left(\lambda_{2}-\lambda_{1}\right)}\left[\int_{-\infty}^{\xi} e^{\lambda_{1}(\xi-s)}+\int_{\xi}^{\infty} e^{\lambda_{2}(\tilde{\xi}-s)}\right]\left[\beta \varphi_{1}(s)+H_{1}\left(\varphi_{1}, \varphi_{2}\right)(s)\right] d s, \\
P_{2}\left(\varphi_{1}, \varphi_{2}\right)(\xi)=\frac{1}{d_{2}\left(\lambda_{4}-\lambda_{3}\right)}\left[\int_{-\infty}^{\xi} e^{\lambda_{3}(\xi-s)}+\int_{\xi}^{\infty} e^{\lambda_{4}(\xi-s)}\right]\left[\beta \varphi_{2}(s)+H_{2}\left(\varphi_{1}, \varphi_{2}\right)(s)\right] d s,
\end{array}\right.
$$

where $\left(\varphi_{1}, \varphi_{2}\right) \in \Sigma$.

Similar to Ma [28] and Lin and Ruan [18], we can prove that $P: \Sigma \rightarrow \Sigma$ is completely continuous in the sense of the decay norm $|\cdot|_{\mu}$ (please see the Appendix A). Therefore, $P$ has a fixed point in $\Sigma$. Denote the fixed point by $\left(\varphi_{1}^{*}, \varphi_{2}^{*}\right)$, it is clear that $\left(\varphi_{1}^{*}, \varphi_{2}^{*}\right)$ satisfies Equation (11) and is strictly positive. The proof is complete.

Theorem 3. Assume that $c>c_{0}, b_{1} \bar{k}_{1}<1$ and $b_{2} \bar{k}_{2}<1$ hold. Then, Equations (11) and (12) have a strictly positive solution, which is a desired traveling wave solution of Equation (4).

Proof. By what we have done, it suffices to verify the asymptotic behavior of Equation (12). We now prove it by the idea in Lin and Ruan ([18], Section 3). We first prove that

$$
\liminf _{\tilde{\xi} \rightarrow \infty} \varphi_{1}(\xi)>0, \liminf _{\tilde{\xi} \rightarrow \infty} \varphi_{2}(\xi)>0
$$

In fact, $\varphi_{1}(\xi)$ satisfies

$$
d_{1} \varphi_{1}^{\prime \prime}(\xi)-c \varphi_{1}^{\prime}(\xi)+r_{1} \varphi_{1}(\xi)\left[1-b_{1} \bar{k}_{1}-a_{1} \varphi_{1}(\xi)\right] \leq 0 .
$$

Let $w(x, t)=\varphi_{1}(x+c t)$, then

$$
\left\{\begin{array}{l}
\frac{\partial w(x, t)}{\partial t} \geq d_{1} \Delta w(x, t)+r_{1} w(x, t)\left[1-b_{1} \bar{k}_{1}-a_{1} w(x, t)\right] \\
w(x, 0)=\varphi_{1}(x)
\end{array}\right.
$$

By the theory of asymptotic spreading (see Aronson and Weinberger [27]) and the basic theory of reaction-diffusion equations (see Ye et al. [29]), if $\varphi_{1}(0)>0$, then

$$
\liminf _{t \rightarrow \infty} w(0, t) \geq\left(1-b_{1} \bar{k}_{1}\right) / a_{1} .
$$


By the invariant form of traveling wave solutions, we obtain

$$
\liminf _{\xi \rightarrow \infty} \varphi_{1}(\xi) \geq\left(1-b_{1} \bar{k}_{1}\right) / a_{1}>0
$$

Similarly, we have

$$
\liminf _{\xi \rightarrow \infty} \varphi_{2}(\xi) \geq\left(1-b_{2} \bar{k}_{2}\right) / a_{2}>0
$$

Define

$$
\liminf _{\tilde{\xi} \rightarrow \infty} \varphi_{i}(\xi)=\varphi_{i}^{-}, \limsup _{\xi \rightarrow \infty} \varphi_{i}(\xi)=\varphi_{i}^{+}, i=1,2 .
$$

Then, there exists $s_{0} \in(0,1)$ such that

$$
E\left(s_{0}\right) \leq\left(\varphi_{1}^{-}, \varphi_{1}^{-}\right) \leq\left(\varphi_{1}^{+}, \varphi_{1}^{+}\right) \leq F\left(s_{0}\right) .
$$

Because $E, F$ are continuous functions on bounded interval, if Equation (12) does not hold, then there exists $s_{0} \in(0,1)$ such that at least one of the following is true

$$
\varphi_{i}^{-}=s_{0} k_{i}, \varphi_{i}^{+}=s_{0} k_{i}+\left(1-s_{0}\right)\left(\bar{k}_{i}+\epsilon_{i}\right), i=1,2 .
$$

If $\varphi_{1}^{-}=s_{0} k_{1}$, then there exists $\left\{\xi_{m}\right\}_{m \in \mathbb{N}}$ with $\lim _{m \rightarrow \infty} \xi_{m}=\infty$ such that

$$
\liminf _{\xi \rightarrow \infty} \varphi_{1}(\xi)=s_{0} k_{1}
$$

and

$$
\liminf _{m \rightarrow \infty}\left(d_{1} \varphi_{1}^{\prime \prime}\left(\xi_{m}\right)-c \varphi_{1}^{\prime}\left(\xi_{m}\right)\right) \geq 0
$$

At the same time, the verification of contracting rectangle implies that

$$
\liminf _{m \rightarrow \infty} H_{1}\left(\varphi_{1}, \varphi_{2}\right)\left(\xi_{m}\right)>0
$$

and a contradiction occurs because $\left(\varphi_{1}, \varphi_{2}\right)$ is a solution for all $\xi \in \mathbb{R}$. In a similar way, we can verify that

$$
E(s) \ll\left(\varphi_{1}^{-}, \varphi_{1}^{-}\right) \leq\left(\varphi_{1}^{+}, \varphi_{1}^{+}\right) \ll F(s) \text { for all } s \in(0,1),
$$

and Equation (12) is true. The proof is complete.

Remark 3. It is possible that $c_{0}=\max \left\{2 \sqrt{d_{1} r_{1}}, 2 \sqrt{d_{2} r_{2}}\right\}$. If $c_{0}=\max \left\{2 \sqrt{d_{1} r_{1}}, 2 \sqrt{d_{2} r_{2}}\right\}$ (e.g., $d_{1} \geq d_{2}$ and $r_{1} \geq r_{2}$ ), then it is the threshold such that Equations (11) and (12) have a positive solution. Very likely, $c_{0}$ is the spreading speed of some unknown functions (see [30,31] for a conclusion in predator-prey system).

Author Contributions: X.-S.L. analyzed and prepared the draft. S.P. analyzed and edited the manuscript. Both authors read the final manuscript.

Funding: This research was funded by NSF of China grant number 11761044.

Acknowledgments: The authors would like to thank the two referees for their careful reading.

Conflicts of Interest: The authors declare no conflict of interest.

\section{Appendix A}

Lemma A1. $P: \Sigma \rightarrow \Sigma$ is completely continuous in the sense of the decay norm $|\cdot| \mu$. 
Proof. The proof is similar to that in [32-35]. We first verify that $P: \Sigma \rightarrow \Sigma$. Let $\xi_{1}$ be a constant such that

$$
e^{\gamma_{1} \xi_{1}}+q \bar{k}_{1} e^{\gamma \xi_{1}}=\bar{k}_{1}
$$

then

$$
\varphi_{1}^{\prime}\left(\xi_{1}^{-}\right)>\varphi_{1}^{\prime}\left(\xi_{1}^{+}\right)=0 .
$$

If $\xi \in\left(-\infty, \xi_{1}\right)$ and $\left(\varphi_{1}, \varphi_{2}\right) \in \Sigma$, then

$$
\begin{aligned}
P_{1}\left(\varphi_{1}, \varphi_{2}\right)(\xi)= & \frac{1}{d_{1}\left(\lambda_{2}-\lambda_{1}\right)}\left[\int_{-\infty}^{\xi} e^{\lambda_{1}(\xi-s)}+\int_{\xi}^{\infty} e^{\lambda_{2}(\xi-s)}\right]\left[\beta \varphi_{1}(s)+H_{1}\left(\varphi_{1}, \varphi_{2}\right)(s)\right] d s \\
\leq & \frac{1}{d_{1}\left(\lambda_{2}-\lambda_{1}\right)}\left[\int_{-\infty}^{\xi} e^{\lambda_{1}(\xi-s)}+\int_{\xi}^{\infty} e^{\lambda_{2}(\xi-s)}\right]\left[\beta \bar{\varphi}_{1}(s)+\bar{H}_{1}(s)\right] d s \\
= & \frac{1}{d_{1}\left(\lambda_{2}-\lambda_{1}\right)}\left[\int_{-\infty}^{\xi} e^{\lambda_{1}(\xi-s)}+\int_{\xi}^{\xi_{1}} e^{\lambda_{2}(\xi-s)}\right]\left[\beta \bar{\varphi}_{1}(s)+\bar{H}_{1}(s)\right] d s \\
& +\frac{1}{d_{1}\left(\lambda_{2}-\lambda_{1}\right)} \int_{\xi_{1}}^{\infty} e^{\lambda_{2}(\xi-s)}\left[\beta \bar{\varphi}_{1}(s)+\bar{H}_{1}(s)\right] d s \\
\leq & \frac{1}{d_{1}\left(\lambda_{2}-\lambda_{1}\right)}\left[\int_{-\infty}^{\xi} e^{\lambda_{1}(\xi-s)}+\int_{\tilde{\xi}}^{\xi_{1}} e^{\lambda_{2}(\xi-s)}\right]\left[\beta \bar{\varphi}_{1}(s)+c \bar{\varphi}_{1}^{\prime}(s)-d_{1} \bar{\varphi}_{1}^{\prime \prime}(s)\right] d s \\
& +\frac{1}{d_{1}\left(\lambda_{2}-\lambda_{1}\right)} \int_{\xi_{1}}^{\infty} e^{\lambda_{2}(\xi-s)}\left[\beta \bar{\varphi}_{1}(s)+c \bar{\varphi}_{1}^{\prime}(s)-d_{1} \bar{\varphi}_{1}^{\prime \prime}(s)\right] d s \\
= & \bar{\varphi}_{1}(\xi)+\frac{e^{\lambda_{2}\left(\xi-\xi_{1}\right)}}{\lambda_{2}-\lambda_{1}}\left[\bar{\varphi}_{1}^{\prime}\left(\xi_{1}^{+}\right)-\bar{\varphi}_{1}^{\prime}\left(\xi_{1}^{-}\right)\right] \\
\leq & \bar{\varphi}_{1}(\xi) .
\end{aligned}
$$

Here, we use the fact

$$
\begin{aligned}
& \frac{d}{d s}\left[e^{-\lambda_{i} s}\left(\left(c-d_{1} \lambda_{i}\right) \phi(s)-d_{1} \phi^{\prime}(s)\right)\right] \\
= & e^{-\lambda_{i} s}\left[-\lambda_{i}\left(c-d_{1} \lambda_{i}\right) \phi(s)+d_{1} \lambda_{i} \phi^{\prime}(s)\right] \\
& +e^{-\lambda_{i} s}\left(\left(c-d_{1} \lambda_{i}\right) \phi^{\prime}(s)-d_{1} \phi^{\prime \prime}(s)\right) \\
= & e^{-\lambda_{i} s}\left[-d_{1} \phi^{\prime \prime}(s)+c \phi^{\prime}(s)+\beta \phi(s)\right], i=1,2,
\end{aligned}
$$

if $\phi(s)$ is twice differentiable.

By a similar recipe, we have

$$
P_{1}\left(\varphi_{1}, \varphi_{2}\right)(\xi) \leq \bar{\varphi}_{1}(\xi)+\frac{e^{\lambda_{1}\left(\xi-\xi_{1}\right)}}{\lambda_{2}-\lambda_{1}}\left[\bar{\varphi}_{1}^{\prime}\left(\xi_{1}^{+}\right)-\bar{\varphi}_{1}^{\prime}\left(\xi_{1}^{-}\right)\right] \leq \bar{\varphi}_{1}(\xi)
$$

if $\xi>\xi_{1}$ and $\left(\varphi_{1}, \varphi_{2}\right) \in \Sigma$. Due to the continuity of $P_{1}\left(\varphi_{1}, \varphi_{2}\right)(\xi), \varphi_{1}(\xi)$, we have

$$
P_{1}\left(\varphi_{1}, \varphi_{2}\right)(\xi) \leq \bar{\varphi}_{1}(\xi), \xi \in \mathbb{R}
$$

Similarly, we have

$$
\underline{\varphi}_{1}(\xi) \leq P_{1}\left(\varphi_{1}, \varphi_{2}\right)(\xi) \leq \bar{\varphi}_{1}(\xi), \underline{\varphi}_{2}(\xi) \leq P_{2}\left(\varphi_{1}, \varphi_{2}\right)(\xi) \leq \bar{\varphi}_{2}(\xi)
$$

if $\xi \in \mathbb{R}$ and $\left(\varphi_{1}, \varphi_{2}\right) \in \Sigma$, and so $P: \Sigma \rightarrow \Sigma$. 
Assume that $\left(\varphi_{1}, \varphi_{2}\right),\left(\varphi_{3}, \varphi_{4}\right) \in \Sigma$, then

$$
\begin{aligned}
& e^{-\mu|s|}\left|\beta \varphi_{1}(s)+H_{1}\left(\varphi_{1}, \varphi_{2}\right)(s)-\left[\beta \varphi_{3}(s)+H_{1}\left(\varphi_{3}, \varphi_{4}\right)(s)\right]\right| \\
\leq & \left(\beta+r_{1}+2 r_{1} a_{1} \bar{k}_{1}\right) e^{-\mu|s|}\left|\varphi_{1}(s)-\varphi_{3}(s)\right| \\
& +e^{-\mu|s|} r_{1} b_{1}\left|\varphi_{1}(s) \int_{-\tau}^{0} \varphi_{1}(s+c t) d \eta_{11}(t)-\varphi_{3}(s) \int_{-\tau}^{0} \varphi_{3}(s+c t) d \eta_{11}(t)\right| \\
& +e^{-\mu|s|} r_{1} e_{1}\left|\varphi_{1}(s) \int_{-\tau}^{0} \varphi_{2}(s+c t) d \eta_{11}(t)-\varphi_{3}(s) \int_{-\tau}^{0} \varphi_{4}(s+c t) d \eta_{11}(t)\right| \\
\leq \quad & \left(\beta+r_{1}+2 r_{1} a_{1} \bar{k}_{1}\right)\left|\left(\varphi_{1}, \varphi_{2}\right)-\left(\varphi_{3}, \varphi_{4}\right)\right|_{\mu} \\
& +r_{1} b_{1} \bar{k}_{1} e^{-\mu|s|}\left\{\left|\varphi_{1}(s)-\varphi_{3}(s)\right|+\left|\int_{-\tau}^{0} \varphi_{1}(s+c t) d \eta_{11}(t)-\int_{-\tau}^{0} \varphi_{3}(s+c t) d \eta_{11}(t)\right|\right\} \\
& +r_{1} e_{1} \bar{k}_{1} e^{-\mu|s|}\left|\varphi_{1}(s)-\varphi_{3}(s)\right| \\
& +r_{1} e_{1} \bar{k}_{2} e^{-\mu|s|}\left|\int_{-\tau}^{0} \varphi_{2}(s+c t) d \eta_{11}(t)-\int_{-\tau}^{0} \varphi_{4}(s+c t) d \eta_{11}(t)\right| \\
\leq \quad & \left(\beta+r_{1}+2 r_{1} a_{1} \bar{k}_{1}\right)\left|\left(\varphi_{1}, \varphi_{2}\right)-\left(\varphi_{3}, \varphi_{4}\right)\right|_{\mu} \\
& +r_{1} b_{1} \bar{k}_{1}\left|\left(\varphi_{1}, \varphi_{2}\right)-\left(\varphi_{3}, \varphi_{4}\right)\right|_{\mu} \\
& +r_{1} b_{1} \bar{k}_{1} e^{\mu c \tau}\left|\int_{-\tau}^{0} e^{-\mu|s+c t|}\left[\varphi_{1}(s+c t)-\varphi_{3}(s+c t)\right] d \eta_{11}(t)\right| \\
& +r_{1} e_{1} \bar{k}_{1}\left|\left(\varphi_{1}, \varphi_{2}\right)-\left(\varphi_{3}, \varphi_{4}\right)\right|_{\mu} \\
& +r_{1} e_{1} \bar{k}_{2} e^{\mu c \tau}\left|\int_{-\tau}^{0} e^{-\mu|s+c t|}\left[\varphi_{2}(s+c t)-\varphi_{4}(s+c t)\right] d \eta_{11}(t)\right| \\
\leq & L\left(\varphi_{1}, \varphi_{2}\right)-\left.\left(\varphi_{3}, \varphi_{4}\right)\right|_{\mu},
\end{aligned}
$$

where $L>0$ is defined by

$$
L=\beta+r_{1}+2 r_{1} a_{1} \bar{k}_{1}+r_{1} b_{1} \bar{k}_{1}+r_{1} b_{1} \bar{k}_{1} e^{\mu c \tau}+r_{1} e_{1} \bar{k}_{1}+r_{1} e_{1} \bar{k}_{2} e^{\mu c \tau} .
$$

By the above estimation, we have

$$
\begin{aligned}
& \left|P_{1}\left(\varphi_{1}, \varphi_{2}\right)(\xi)-P_{1}\left(\varphi_{3}, \varphi_{4}\right)(\xi)\right| e^{-\mu|\xi|} \\
= & \frac{e^{-\mu|\xi|}}{d_{1}\left(\lambda_{2}-\lambda_{1}\right)} \mid\left[\int_{-\infty}^{\xi} e^{\lambda_{1}(\xi-s)}+\int_{\xi}^{\infty} e^{\lambda_{2}(\xi-s)}\right] e^{\mu|s|} e^{-\mu|s|}\left[\beta \varphi_{1}(s)+H_{1}\left(\varphi_{1}, \varphi_{2}\right)(s)\right] d s \\
& -\left[\int_{-\infty}^{\xi} e^{\lambda_{1}(\xi-s)}+\int_{\xi}^{\infty} e^{\lambda_{2}(\xi-s)}\right] e^{\mu|s|} e^{-\mu|s|}\left[\beta \varphi_{3}(s)+H_{1}\left(\varphi_{3}, \varphi_{4}\right)(s)\right] d s \mid \\
\leq & \frac{L e^{-\mu|\xi|}\left|\left(\varphi_{1}, \varphi_{2}\right)-\left(\varphi_{3}, \varphi_{4}\right)\right|_{\mu}}{d_{1}\left(\lambda_{2}-\lambda_{1}\right)}\left[\int_{-\infty}^{\xi} e^{\lambda_{1}(\xi-s)}+\int_{\xi}^{\infty} e^{\lambda_{2}(\xi-s)}\right] e^{\mu|s|} d s \\
\leq & \frac{L\left|\left(\varphi_{1}, \varphi_{2}\right)-\left(\varphi_{3}, \varphi_{4}\right)\right|_{\mu}}{d_{1}\left(\lambda_{2}-\lambda_{1}\right)}\left[\int_{-\infty}^{\xi} e^{\left(\lambda_{1}+\mu\right)(\xi-s)}+\int_{\xi}^{\infty} e^{\left(\lambda_{2}-\mu\right)(\xi-s)}\right] e^{\mu|s|} d s \\
= & \frac{L\left|\left(\varphi_{1}, \varphi_{2}\right)-\left(\varphi_{3}, \varphi_{4}\right)\right|_{\mu}}{d_{1}\left(\lambda_{2}-\lambda_{1}\right)}\left[\frac{1}{\lambda_{2}-\mu}-\frac{1}{\lambda_{1}+\mu}\right],
\end{aligned}
$$

and so

$$
\sup _{\xi \in \mathbb{R}}\left\{\left|P_{1}\left(\varphi_{2}, \varphi_{2}\right)(\xi)-P_{1}\left(\varphi_{3}, \varphi_{4}\right)(\xi)\right| e^{-\mu|\xi|}\right\} \leq \frac{L\left|\left(\varphi_{1}, \varphi_{2}\right)-\left(\varphi_{3}, \varphi_{4}\right)\right|_{\mu}}{d_{1}\left(\lambda_{2}-\lambda_{1}\right)}\left[\frac{1}{\lambda_{2}-\mu}-\frac{1}{\lambda_{1}+\mu}\right] .
$$

By a similar argument on $P_{2}$, we see that $P: \Sigma \rightarrow \Sigma$ is continuous in the sense of $|\cdot|_{\mu}$. 
We now prove that $P: \Sigma \rightarrow \Sigma$ is compact in the sense of $|\cdot|_{\mu}$. For any given $\epsilon>0$, there exists a constant $N>0$ such that

$$
e^{-\mu N}\left(\bar{k}_{1}+\bar{k}_{2}\right)<\epsilon .
$$

Since $\beta \varphi_{i}(s)+H_{i}\left(\varphi_{1}, \varphi_{2}\right)(s), i=1,2$, are bounded and continuous in $s \in \mathbb{R}$, we see that

$$
\begin{aligned}
& \left|\frac{d}{d \xi} P_{1}\left(\varphi_{1}, \varphi_{2}\right)(\xi)\right| \\
= & \left|\frac{1}{d_{1}\left(\lambda_{2}-\lambda_{1}\right)}\left[\int_{-\infty}^{\xi} \lambda_{1} e^{\lambda_{1}(\xi-s)}+\int_{\xi}^{\infty} \lambda_{2} e^{\lambda_{2}(\xi-s)}\right]\left[\beta \varphi_{1}(s)+H_{1}\left(\varphi_{1}, \varphi_{2}\right)(s)\right] d s\right|
\end{aligned}
$$

and

$$
\begin{aligned}
& \left|\frac{d}{d \xi} P_{2}\left(\varphi_{1}, \varphi_{2}\right)(\xi)\right| \\
= & \left|\frac{1}{d_{2}\left(\lambda_{4}-\lambda_{3}\right)}\left[\int_{-\infty}^{\xi} \lambda_{3} e^{\lambda_{3}(\xi-s)}+\int_{\xi}^{\infty} \lambda_{4} e^{\lambda_{4}(\xi-s)}\right]\left[\beta \varphi_{2}(s)+H_{2}\left(\varphi_{1}, \varphi_{2}\right)(s)\right] d s\right|
\end{aligned}
$$

are uniformly bounded. By Ascoli-Arzela lemma, when we restrict $P(\Sigma)$ on $[-N, N]$, we have a finite $\epsilon-$ net. By Equation (A1), the net is also a finite $\epsilon-$ net of $P(\Sigma)$ in the sense of $|\cdot|_{\mu}$. The proof is complete.

\section{References}

1. Boucher, D.H. The Biology of Mutualism: Ecology and Evolution; Croom Helm: London, UK, 1985.

2. Malchow, H.; Petrovskii, S.V.; Venturino, E. Spatiotemporal Patterns in Ecology and Epidemiology: Theory, Models and Simulatio; Chapman \& Hall/CRC: Boca Raton, FL, USA, 2008.

3. Bianca, C.; Pappalardo, F.; Pennisi, M.; Ragusa, M.A. Persistence analysis in a Kolmogorov-type model for cancer-immune system competition. AIP Conf. Proc. 2013, 1558, 1797-1800.

4. Ragusa, M.A.; Russo, G. ODEs approaches in modeling fibrosis. Comment on “Towards a unified approach in the modeling of fibrosis: A review with research perspectives" by Martine Ben Amar and Carlo Bianca. Phys. Life Rev. 2016, 17, 112-113. [CrossRef] [PubMed]

5. Smith, H.L. Monotone Dynamical Systems: An Introduction to the Theory of Competitive and Cooperative Systems; AMS: Providence, RI, USA, 1995.

6. Li, B.; Weinberger H.F.; Lewis, M.A. Spreading speeds as slowest wave speeds for cooperative systems. Math. Biosci. 2005, 196, 82-98. [CrossRef] [PubMed]

7. Lin, G.; Li, W.T.; Ruan, S. Monostable wavefronts in cooperative Lotka-Volterra systems with nonlocal delays. Discrete Cont. Dyn. Syst. 2011, 31, 1-23. [CrossRef]

8. Lin, G. Asymptotic spreading fastened by inter-specific coupled nonlinearities: A cooperative system. Physica D 2012, 241, 705-710. [CrossRef]

9. Huang, J.; Zou, X. Traveling wave fronts in diffusive and cooperative Lotka-Volterra system with delays. J. Math. Anal. Appl. 2002, 271, 455-466. [CrossRef]

10. Li, W.T.; Wang, Z.C. Traveling fronts in diffusive and cooperative Lotka-Volterra system with non-local delays. Z. Angew. Math. Phys. 2007, 58, 571-591. [CrossRef]

11. Li, X.; Lin, G. Traveling wavefronts in nonlocal dispersal and cooperative Lotka-Volterra system with delays. Appl. Math. Comput. 2008, 204, 738-744. [CrossRef]

12. Lin, G.; Li, W.T.; Ma, M. Travelling wave solutions in delayed reaction diffusion systems with applications to multi-species models. Discrete Cont. Dyn. Syst. Ser. B 2010, 19, 393-414.

13. Wang, H.; Castillo-Chavez, C. Spreading speeds and traveling waves for non-cooperative integro-difference systems. Discrete Cont. Dyn. Syst. Ser. B 2012, 17, 2243-2266. [CrossRef] [PubMed]

14. Yi, T.; Chen, Y.; $\mathrm{Wu}, \mathrm{J}$. Unimodal dynamical systems: Comparison principles, spreading speeds and travelling waves. J. Differ. Equ. 2013, 254, 3538-3572. [CrossRef]

15. Fang, J.; Zhao, X.Q. Existence and uniqueness of traveling waves for non-monotone integral equations with applications. J. Differ. Equ. 2010, 248, 2199-2226. [CrossRef] 
16. Ma, S. Traveling waves for non-local delayed diffusion equations via auxiliary equations. J. Differ. Equ. 2007, 237, 259-277. [CrossRef]

17. Martin, R.H.; Smith, H.L. Reaction-diffusion systems with the time delay: Monotonicity, invariance, comparison and convergence. J. Reine Angew. Math. 1991, 413, 1-35.

18. Lin, G.; Ruan, S. Traveling wave solutions for delayed reaction-diffusion systems and applications to Lotka-Volterra competition-diffusion models with distributed delays. J. Dyn. Differ. Equ. 2014, 26, 583-605. [CrossRef]

19. Meng, Y.; Zhang, W.; Yu, Z. Existence and asymptotic of traveling wave fronts for the delayed Volterra-type cooperative system with spatial diffusion. Adv. Differ. Equ. 2018, 2018, 203. [CrossRef]

20. Wang, Z.C.; Li, W.T. Dynamics of a non-local delayed reaction-diffusion equation without quasi-monotonicity. Proc. R. Soc. Edinb. Sect. A 2010, 140, 1081-1109. [CrossRef]

21. Wu, S.L.; Hsu, C.H. Entire solutions of non-quasi-monotone delayed reaction-diffusion equations with applications. Proc. R. Soc. Edinb. Sect. A 2014, 144, 1085-1112. [CrossRef]

22. Zhang, L.; Pan, S. Entire solutions of integrodifference equations. J. Differ. Equ. Appl. 2019. [CrossRef]

23. Faria, T. Sharp conditions for global stability of Lotka-Volterra systems with distributed delays. J. Differ. Equ. 2009, 246, 4391-4404. [CrossRef]

24. Faria, T. Stability and extinction for Lotka-Volterra systems with infinite delay. J. Dyn. Differ. Equ. 2010, 22, 299-324. [CrossRef]

25. Hale, J.K.; Verduyn Lunel, S.M. Introduction to Functional-Differential Equations; Springer-Verlag: New York, NY, USA, 1993.

26. Martin, R.H.; Smith, H.L. Abstract functional differential equations and reaction-diffusion systems. Trans. Am. Math. Soc. 1990, 321, 1-44.

27. Aronson, D.G.; Weinberger, H.F. Nonlinear diffusion in population genetics, combustion, and nerve pulse propagation. In Partial Differential Equations and Related Topics; Goldstein, J.A., Ed.; Lecture Notes in Mathematics; Springer: Berlin, Germany, 1975; Volume 446, pp. 5-49.

28. Ma, S. Traveling wavefronts for delayed reaction-diffusion systems via a fixed point theorem. J. Differ. Equ. 2001, 171, 294-314. [CrossRef]

29. Ye, Q.; Li, Z.; Wang, M.; Wu, Y. Introduction to Reaction-Diffusion Equations; Science Press: Beijing, China, 2011.

30. Ducrot, A. Spatial propagation for a two component reaction-diffusion system arising in population dynamics. J. Differ. Equ. 2016, 260, 8316-8357. [CrossRef]

31. Pan, S. Invasion speed of a predator-prey system. Appl. Math. Lett. 2017, 74, 46-51. [CrossRef]

32. Huang, J.; Zou, X. Existence of traveling wavefronts of delayed reaction-diffusion systems without monotonicity. Discrete Cont. Dyn. Syst. 2003, 9, 925-936. [CrossRef]

33. Li, W.T.; Lin, G; Ruan, S. Existence of traveling wave solutions in delayed reaction-diffusion systems with applications to diffusion-competition systems. Nonlinearity 2006, 19, 1253-1273. [CrossRef]

34. Wang, Z.C.; Li, W.T.; Ruan, S. Traveling wave fronts of reaction-diffusion systems with spatio-temporal delays. J. Differ. Equ. 2006, 222, 185-232. [CrossRef]

35. Wu, J.; Zou, X. Traveling wave fronts of reaction-diffusion systems with delay. J. Dyn. Differ. Equ. 2001, 13, 651-687. [CrossRef]

(C) 2019 by the authors. Licensee MDPI, Basel, Switzerland. This article is an open access article distributed under the terms and conditions of the Creative Commons Attribution (CC BY) license (http://creativecommons.org/licenses/by/4.0/). 